\title{
OBSERVACIONES SOBRE ALGUNOS ASPECTOS DE LA FILOSOFÍA DE ERNST TUGENDHAT
}

\author{
David MarTínez Rincón* \\ doi: 10.11144/Javeriana.uph35-71.oaft
}

\section{RESUMEN}

En este artículo, quiero proponer la tesis de que hay un hilo conductor en la filosofía de Tugendhat que vincula el análisis conceptual con su teoría moral y su teoría de la justicia. Mi intención es reconstruir algunos aspectos de su filosofía siguiendo su tesis de que es necesaria una aclaración conceptual para explicar qué es la justicia. Para tal fin, divido el siguiente texto en cuatro partes: en la primera, expongo qué entiende el autor por aclaración o análisis conceptual; en la segunda, explico algunos rasgos de su teoría moral, el contractualismo; y, en la tercera, presento su teoría de la justicia. Finalmente, doy cuenta de algunas conclusiones que enmarcan mi trabajo en el estudio de la discusión de la teoría de la justicia de Rawls y Tugendhat.

Palabras clave: teoría de la justicia; contractualismo; igualdad; bueno; deber

Universidad de Antioquia, Medellín, Colombia.

Correo electrónico: eldavidmartinez@gmail.com

Para citar este artículo: Martínez Rincón, D. (2018). Observaciones sobre algunos aspectos de la filosofía de Ernst Tugendhat. Universitas Philosophica, 35(71), pp. 351-377. ISSN 0120-5323, ISSN en línea 2346-2426. doi: 10.11144/Javeriana.uph35-71.oaft 


\title{
REMARKS ABOUT SOME ASPECTS OF THE PHILOSOPHY OF ERNST TUGENDHAT
}

\begin{abstract}
This paper argues that there is a thread in the philosophy of E. Tugendhat binding together his moral and justice theories with his conceptual analysis. Oriented by the thesis that a conceptual analysis is necessary to explain what justice is, this paper reconstructs some aspects of Tugendhat's philosophy. It is divided in four sections: First, I delve into the author's definition and use of conceptual analysis. Second, I describe some features of Tugendhat's moral theory of contractualism. Third, I examine his theory of justice. Fourth, I conclude by framing my analysis within the debate between Rawls's and Tugendhat's theories of justice.
\end{abstract}

Keywords: theory of justice; contractualism; equality; good; duty 
EN EL SIGLO XX, dos corrientes metodológicas en la investigación de la filosofía, la fenomenología y la filosofía analítica del lenguaje dieron lugar a trabajos en metodología de la filosofía como Introducción a la filosofía analítica (2003) ${ }^{1}$ de Tugendhat, Las investigaciones fllosóficas (1999) de Wittgenstein o la clásica recopilación de ensayos de Rorty El giro lingüistico (1967). La propuesta metodológica de Tugendhat es particularmente sobresaliente porque orienta sus reflexiones sobre el método a la filosofía práctica. Su exposición del análisis del lenguaje no es solo un elemento propedéutico. Cumple la función de darle sentido al análisis semántico de sus problemas morales y políticos.

A principio del siglo XX, originalmente acuñada por el comentarista Bergmann, surge la expresión giro lingüístico, más tarde popularizada en la compilación de ensayos sobre la filosofía del lenguaje editada por Rorty (1967, p. 9). El compilador afirma que el giro lingüístico, que también recibe el nombre de filosofía del lenguaje, es uno de los más importantes "descubrimientos filosóficos de nuestro tiempo y quizá de nuestra época” (p. 9), y explica que por filosofía de lenguaje se entiende "la perspectiva según la cual los problemas filosóficos son problemas que pueden resolverse (o disolverse) al reformular el lenguaje o al entender más sobre el lenguaje que usamos en el presente" (p. 3). Lo que se designa como giro lingüistico consiste en poner el lenguaje en el centro de la reflexión filosófica. Tugendhat se encuentra entre los autores que toman partido por esta reflexión sobre el lenguaje. La afirmación de Rorty es equivalente a la afirmación de Tugendhat (2003) en la que define la filosofía analítica del lenguaje: "está

1 La obra citada es una traducción parcial castellana de Vorlesungen zur Einführung in die sprachanalytische Philosophie (1976). Existe una traducción al inglés completa de la obra alemana denominada Traditional and Analytical Philosophy. Lectures on the Philosophy of Language (1982). Dado que la versión castellana es solo una traducción de la primera parte de la obra, en adelante para citas específicas me referiré al texto inglés. 
claro que al hablar de 'filosofía analítica del lenguaje' nos referimos a una manera de filosofar que cree poder o debe resolver los problemas planteados a la filosofía por el camino de un análisis del lenguaje" (p. 18).

Su iniciación en la filosofía analítica del lenguaje tiene lugar en los tres primeros años como catedrático en la Universidad de Heidelberg, luego de haber pasado, en 1965, una temporada en los Estados Unidos, que consideró como una verdadera "revolución mental" (Tugendhat, 1998, p. 12). El autor escribe algunos artículos sobre filosofía analítica del lenguaje en su semestre sabático y luego publica, en 1976, el que puede considerarse su texto más importante sobre filosofía analítica del lenguaje traducido al castellano como Introducción a la filosofía analítica. La intención del texto es reflexionar sobre los propios fundamentos de la filosofía analítica que son una condición para llevar a cabo la tarea filosófica de examinar las preguntas, los métodos y los conceptos fundamentales dados, así como para elaborar preguntas, métodos y conceptos fundamentales nuevos ( $\mathrm{Tu}-$ gendhat, 2003, p. 13). Además, el autor quiere mostrar allí que la filosofía analítica otorga un significado concreto y realizable a la pregunta heideggeriana por el ser.

Tugendhat no se pronuncia explícitamente sobre el giro lingüístico aun cuando este sea el contexto que lo enmarca. Los estudiosos, ciertamente, se refieren a la relación entre el giro lingüístico y el pensamiento de Tugendhat (Zabala, 2005 y Bartolucci, 2008) y, en su obra, Tugendhat sí critica la comprensión tradicional del lenguaje como representación del mundo, privilegiando un trabajo filosófico sobre el lenguaje que atiende a sus usos y a las reglas, explícitas o implícitas, que los organizan. La versión tradicional del lenguaje consiste en que las expresiones lingüísticas adquieren significado gracias a que representan objetos (Tugendhat, 1982, pp. 96 y ss., y 1997a, pp. 73 y ss.). Esta comprensión puede entenderse a partir de enunciados del tipo "esta mesa es café". Mientras el yo está aquí con sus afirmaciones como "esta mesa es café", el mundo está allá, y es lo nombrado por el lenguaje, en este caso la mesa de cuatro patas que percibimos con los ojos, y sirve para sostener nuestros libros y apoyarnos en ella. El verbo ser representa algo del mundo, el ser o la esencia de la mesa. Así, la filosofía tradicional explicó el lenguaje como un medio de expresión de la relación del yo con el mundo.

A la idea de que el lenguaje es una representación, y para deshacerse de la dicotomía entre el yo y el mundo, Tugendhat opone la consideración de que el lenguaje adquiere sentido únicamente como conjunto de prácticas sociales. 
El lenguaje así entendido no es sino una forma de conducta, entre otras, que tiene innumerables funciones: ordenar, describir, informar, hacer conjeturas, contar historias, hacer teatro, contar chistes y adivinar enigmas, entre otras.

El superar una comprensión negativa y crítica del lenguaje como representación y acoger aquella positiva que lo concibe en función de sus usos, le permite a Tugendhat avanzar en la adopción del método de clarificación del lenguaje. Se trata de una idea orientadora que el autor toma del último Wittgenstein, para quien este método consiste en describir las reglas de uso de una expresión. Hereda así del pensador austriaco la idea de que es necesario aclarar el lenguaje para examinar problemas filosóficos, aunque redefiniendo su método como un trabajo de "clarificación del significado" de una palabra (Tugendhat, 1984, p. 50), y denominándolo de distintos modos: método analítico, semántica formal, acceso semántico y semántica filosófica -que se distingue de la semántica lingüistica-. También usa el término semántica subjetiva, en contraste con una semántica objetiva (Tugendhat, 1998, p. 183). Con cada una de estas denominaciones, Tugendhat destaca rasgos y matices importantes de uno y el mismo método en diferentes contextos.

Llama a su procedimiento método analítico en el contexto de su pertenencia a la tradición de análisis de lenguaje. Emplea la acuñación semántica formal en un contexto en el que discute la pregunta aristotélica del ente en cuanto ente en relación con el análisis del lenguaje (Tugendhat, 2003, pp. 47 y ss.). Crea la acuñación acceso semántico en el contexto de los problemas de la moral (Tugendhat, 1988, pp. 69 y ss.), refiriéndose a la semántica filosófica para resaltar la importancia del análisis filosófico en la investigación sobre el lenguaje (Tugendhat, 1998, p. 182). Finalmente, hace mención de una semántica objetiva para aludir a una investigación del lenguaje en la que se constata la importancia de las expresiones lingüísticas desde el propio lenguaje (Tugendhat, 1998, p. 183). Este no es un recuento exhaustivo de todas las formas en las que se refiere a su método, pero hay un rasgo común que subyace a estas denominaciones que es la aclaración como principio metodológico más general: "la aclaración de los conceptos consiste en una explicación de la utilización de las expresiones lingüísticas" (Tugendhat, 1998, p. 182). O, como lo expresa en otro de sus ensayos: "delimitar un concepto significa la comprensión de determinadas expresiones lingüísticas" (Tugendhat, 1988, p. 69). 
El autor habla de "método analítico del lenguaje", pero es claro que no se trata del concepto de análisis en el sentido de descomponer las distintas formas de subsumir un término genérico en otros más elementales. Tampoco se alude al análisis de nociones para encontrar una más básica o que justifique a las demás. En cambio, puede hablarse de análisis en su método en el sentido de hacer explícitas las relaciones entre diversos conceptos. Dicho de otro modo, el análisis de las expresiones se lleva a cabo estableciendo diferencias y relaciones entre las distintas palabras. Tugendhat emplea la metáfora de una "estructura reticulada" en Ser, verdad y acción (1998, p. 184), para describir el entramado conceptual en que aparece un concepto y en el que adquiere un significado específico.

Aunque la figura de una red presente en la idea de "estructura reticulada" permite entender que lo que hace el método de aclaración es establecer las relaciones entre conceptos, es una metáfora todavía imprecisa. Ursula Wolf (2002) señala que hay un consenso sobre la clarificación lingüística y la concepción del último Wittgenstein "según la cual se trata de conocer la regla de uso de una expresión" (p. 202), tal como se había señalado antes. Esta es, me parece, la comprensión más general que se puede tener cuando se intenta determinar qué significa aclarar conceptos. Sin embargo, Wolf expone lo problemática que puede ser esta idea cuando se aplica a "conceptos fundamentales". Primero, porque no se ha entendido la relación entre las explicaciones de palabras como tiempo con la filosofía y, segundo, porque esto no es lo que de hecho hacen los filósofos formados analíticamente. Wolf (2002) se pregunta entonces qué significa describir la regla de uso de una palabra, indicando que "los conceptos fundamentales [...] señalan puntos en la estructura fundamental de la comprensión, la cual está constituida por una red de conceptos interdependientes" (p. 204)2.

Para lograr una mayor precisión, la explicación de la metáfora "estructura reticulada" puede ser ampliada con los ejemplos de los "dibujos de la vida" presentados en El método analítico (Tugendhat, 1984, p. 54). Es él mismo quien llama la atención sobre estos ejemplos usados por Wittgenstein $(1998$, p. 105) para referirse a la forma en que un niño aprende las reglas de uso de una expresión, por

2 Esto suscita nuevamente dificultades, porque la interdependencia o la relación de los conceptos puede explicarse a partir de varios modelos como el de Davidson, el de Strawson o el de Ryle, cuyas diferencias y relaciones no son tema de este trabajo. 
ejemplo, un conjunto de palabras como cuando, al mismo tiempo o en el momento en que, todas relacionadas con el concepto de tiempo. Tugendhat explica de qué manera Wittgenstein acude a dos tipos de ilustraciones: el primero es un dibujo en el que se presentan distintas posiciones del sol en un paisaje y el segundo es una ilustración con los distintos hábitos de la vida diaria. Con el propósito de explicar la palabra tiempo, el niño debe primero aprender a coordinar ambos dibujos, de modo que sepa, por ejemplo, relacionar el dibujo de tomar el desayuno con el dibujo en el paisaje donde todavía no ha salido el sol. Luego se le enseña el grupo de palabras antes y después para que aprenda a relacionar ambos dibujos. Así puede formular oraciones como "antes de que el sol salga, el niño toma el desayuno". Relacionar la metáfora de la estructura reticulada con los ejemplos de los dibujos de la vida es la mejor forma de explicar la aclaración de conceptos como un modo de enseñarles a otros el modo de uso de una palabra.

Esta explicación de la estructura reticulada de las expresiones lingüísticas constituye un abandono de la búsqueda de las palabras sustantivas (Tugendhat, 1984, pp. 53-54). Tugendhat critica que pensemos la palabra tiempo como si tuviera un sentido análogo a acero y que creamos que, refiriéndonos a aquel, la pregunta "qué es" tenga sentido (Tugendhat, 1984, p. 54). Si bien podemos explicar la palabra acero buscando una cosa que podemos agarrar, medir o pesar, que nos permita caracterizarla, no podemos explicar palabras como tiempo, idea, espacio o, para lo que nos interesa más adelante, justicia o deber, buscando un referente material que le corresponda.

Para finalizar este apartado, quiero retomar algunos conceptos mencionados que resumen el método de Tugendhat y mostrar cómo los aplica a la justicia. Me refiero al análisis del lenguaje, la descripción, el oponerse a la descomposición de un término, hacer explícitas las relaciones conceptuales, considerar la estructura reticular de un concepto y abandonar las palabras sustantivas. El autor no descompone un término general en los términos que lo componen. Es decir, no toma el concepto de justicia -o de deber o de bueno- como si fuera un término general que se puede descomponer en términos particulares. Su método tampoco consiste en tomar la justicia como una palabra sustantiva y buscar el objeto al que se refiere. Más bien, su propósito es identificar los grupos de palabras vinculados a conceptos como el de la justicia. Hay que destacar, además, que el análisis lingüístico no supone perder contacto con la realidad, pues lleva a cabo una reflexión 
sobre el lenguaje que orienta la elaboración de una pregunta práctica fundamental; pero esta pregunta carecería de sentido en la obra de Tugendhat sin el análisis lingüístico. Así, muestra las relaciones entre los términos justicia, igualdad, deber, bueno, mostrando sus relaciones conceptuales, como veremos a continuación.

\section{La teoría moral de Tugendhat: el contractualismo}

LA IDEA QUE DA ORIGEN a la teoría moral del contractualismo en Tugendhat reside en que no puede haber un fundamento autoritario, tradicional o teológico, ni una verdad superior o trascendental que sustente dicha teoría. ¿Cómo explicar, entonces, la teoría moral sin acudir a la tradición, a la autoridad o apelando a Dios? Tugendhat responde que no se trata de hallar fundamentos últimos, ni siquiera fundamentos, sino justificaciones basadas en razones.

En tal sentido, Tugendhat entiende la moral desde el punto de vista de las exigencias recíprocas. Exigimos no fallar moralmente y esta exigencia es recíproca puesto que todos aceptamos voluntariamente y queremos pertenecer a una comunidad moral que es fuente y destino de la norma. Este tipo de exigencias hallan su justificación en las razones que nos damos unos a otros en una comunidad moral; es decir, su justificación consiste en la aceptación recíproca de dichas razones. En la teoría moral de este autor, como vemos, es decisivo que haya un conjunto de razones debido a las cuales aceptamos las normas de la moral. A la vez, aceptar las normas morales es estar dispuestos a reaccionar con respecto a quien no las cumple con sentimientos de culpa, vergüenza, resentimiento e indignación. Es decir, quien incumple las normas morales, quien las transgrede, experimenta una sanción interna. Tugendhat señala que hay dos tipos fundamentales de sanciones internas: la vergüenza y la culpa.

Sin embargo, las normas morales no pueden ser justificadas desde la perspectiva de cada individuo y, por ello, Tugendhat considera que deben satisfacer un estándar de bien moral que pueda ser reconocido por todos. De allí que considere el contenido del imperativo categórico kantiano y afirme que el sentido de bueno consiste en no instrumentalizar a nadie.

Todo el modelo moral expuesto anteriormente (el conjunto de conceptos morales como deber, razones, sentimientos y bueno) tiene por eje el concepto de deber y con él se pretende introducir, de un modo justificado, el concepto de derecho y, 
en consecuencia, justificar el concepto de derechos humanos. Tugendhat muestra que, así como tenemos deberes en una comunidad moral, también tenemos derechos correspondientes. Esta es la teoría moral planteada de un modo general. Sin embargo, hay algunas diferencias y matices entre los diferentes planteamientos del autor. Su posición evoluciona desde su primera obra sobre ética, Problemas de ética, hasta la última, El origen de la igualdad normativa, dado que es sometida a un proceso de autocrítica. La revisión de sus teorías la hace por vez primera en sus Retractaciones, escritas entre Problemas de ética y Lecciones de ética como respuesta al estudio de Ursula Wolf El problema del deber moral y el texto de Habermas Ética del discurso. Notas sobre un programa de fundamentación ${ }^{3}$. De lo que se retracta en las Retractaciones es del contractualismo de Problemas de ética. Como lo afirma el propio autor en la introducción de Ser, verdad y acción, refiriéndose a las propias retractaciones, estas "hacían el avance esencial de liberarse de la posición contractualista de las tres conferencias" (Tugendhat, 1998, p. 19). Sin embargo, intentaremos mostrar a continuación cómo se conserva cierto tipo de contractualismo -que el autor describe como "simétrico"- y cómo la evolución de este concepto muestra la unidad y la coherencia del pensamiento de Tugendhat.

\subsection{SEMÁNTICA Y MORAL}

La pregunta Central de la due parte Tugendhat en su filosofía moral es por la posibilidad de justificar los juicios morales. Mientras que esta justificación es una necesidad de la vida concreta, hay justificaciones de otro tipo de juicios, como los científicos, que no son apremiantes. La necesidad de justificar los juicios morales reaparece en un momento en el que las convicciones morales pierden su justificación y caen en el relativismo. De manera pues que, cuando se cae en esta relatividad histórica, es necesario justificar los propios juicios morales y esto debe hacerse en relación con los demás. Entendida así, la moral es un conjunto de exigencias recíprocas que deben estar justificadas. Se hace mención de

3 Eriksen (2009) piensa que hay una tendencia en Tugendhat a "exagerar las diferencias que existen entre la diversidad de concepciones de justificación de la moral que él ha dado". Lo que quiere Eriksen es demostrar que Tugendhat "tiene una teoría moral unificada pese a todas las revisiones" (p. 4). 
la reciprocidad porque existe un destino de justificación o alguien a quien se orienta la justificación, que a su vez exige a la fuente o a quien proporciona la justificación un conjunto de acciones y omisiones. Así, Tugendhat propone una estructura intersubjetiva para hablar de la justificación de los juicios morales.

Sin embargo, el acceso semántico a la moral no es algo evidente y puede suscitar preguntas como, por ejemplo, si un análisis de cómo hablamos nos permite decidir entre qué sea bueno y malo. La respuesta que ofrece al cuestionamiento del análisis de las formas en que hablamos es que apelar a la razón práctica, a Dios y a sus mandamientos, o al sentimiento moral, sin un análisis de cómo hablamos y de la experiencia empírica, no proporciona una respuesta satisfactoria. Ninguna de las respuestas anteriores es suficiente porque no proporciona un fundamento para elegir un criterio que nos permita decidir qué sea bueno o qué sea malo. La forma en que se puede fundamentar un criterio para decidir entre qué es bueno y qué es malo es con un acceso semántico, es decir, con una aclaración de la forma en que usamos nuestras expresiones morales. La razón es que la moral se compone inicialmente de expresiones como deber, bueno o moral, cuyo uso debe aclararse. Lo anterior no es solo un modo de acceder a la moral, es además un método para analizar las palabras y proposiciones relevantes del lenguaje moral.

Para comenzar con la aplicación de este método de aclaración, Tugendhat muestra que hay uno o dos conceptos estándar que utilizamos en los juicios morales: bueno y deber. De ahí que concluya que "en nuestra interrogación semántica tenemos que considerar en particular tres palabras: primero la propia palabra moral y, en segundo lugar, determinados usos de las palabras bueno y deber a los que esta palabra parece remitir" (Tugendhat, 1988, p. 75).

El autor hace un análisis de los sentidos ordinarios de la palabra bueno y, además, analiza los usos específicamente morales de esta palabra, estableciendo dos principios importantes: (1) el análisis semántico tiene que afinarse aclarando en principio los usos ordinarios de los términos, pasando a una aclaración de contextos más específicos en los que se hace un uso más determinado de ellos; y (2) en las aclaraciones conceptuales hay un poco de arbitrariedad, de modo que lo importante es lo que queremos decir. Veamos, por ejemplo, cómo aclara el concepto de bueno por medio de expresiones lingüísticas.

El primer uso de la palabra bueno que aclara es cuando la usamos en expresiones como "para adelgazar, es bueno comer menos". Se trata de la relación 
entre medios y fines en la que establecemos medios adecuados para un fin determinado.

Un segundo uso es cuando empleamos la palabra bueno con respecto a algo o alguien. Por ejemplo, "sería bueno un poco de abono para las flores". En este uso, bueno significa lo mismo que conveniente; y malo, sinónimo de perjudicial. Debe considerarse que conveniente y perjudicial son antónimos. La diferencia específica entre el primer uso y el segundo respecto a la palabra bueno reside en que mientras el primero tiene el sentido de algo que propicia un fin determinado, el segundo uso tiene el sentido de propiciar el bienestar propio o de aquello para lo que consideramos que algo es conveniente.

Un tercer uso de la palabra bueno es el atributivo. Lo usamos en expresiones como "un buen cuchillo" o "un buen futbolista". En todos estos casos, bueno parece remitir a una preferencia objetiva, es decir, amparada en razones (Tugendhat, 1988, p. 79). El autor traduce este significado de bueno como preferencia a enunciados de deber. Por ejemplo, el enunciado "este cuchillo es mejor que aquel" implica que debería preferirse por ciertas razones. Esto lo lleva a aclarar los usos del concepto de deber. Su propósito es mostrar que cuando traducimos enunciados con la palabra bueno a enunciados con la palabra deber se quiere señalar que es racional preferir aquello de lo que decimos que es bueno.

Tugendhat continúa analizando si hay usos específicamente morales de bueno y deber. Así, orienta su reflexión hacia el uso predicativo, pues la palabra bueno se predica de algunas acciones. Con frecuencia, se dice que es irracional no obrar de ese modo y hay una vinculación entre lo racional y el uso del término bueno. Sin embargo, Tugendhat no opta por delimitar las proposiciones valorativas a las normas racionales. Esto quiere decir que va a considerar una diferencia entre los juicios de valor y el concepto de razón. Él explica que este concepto tiene un sentido particular, a saber, un sentido metafísico, cuyo examen desarrollará de modo más amplio en trabajos posteriores. Se trata de un sentido que Tugendhat critica para favorecer la teoría de una moral basada en razones que nos damos unos a otros, esto es, una moral desprovista de fundamentación metafísica. Es necesario aclarar en este punto que el autor de ninguna manera quiere apelar a la irracionalidad de la moral ni despojar a la moral de las razones que nos damos en una comunidad moral; por el contrario, soslayar el sentido metafísico de razón le permite acudir a un sentido en el que la razón consiste en explicarnos unos a otros por qué actuamos o elegimos de tal o cual modo. 
Tugendhat explora entonces el uso de la palabra bueno con normas que no son normas racionales, sino normas sociales; es decir, comprende las normas morales a partir de las normas sociales. Lo que no quiere decir que una norma sea aceptada como moral porque la sociedad la refrende, ni tampoco que lo aceptado socialmente sea equivalente a lo moral. Hay muchas normas que la sociedad ha aceptado por consenso y que posteriormente ha rechazado, tras un avance en sus posiciones morales. El punto puede ejemplificarse con la segregación racial y la exclusión de las minorías afrodescendientes, comportamientos que eran aceptables (piénsese en la esclavitud) y que luego pasaron a ser condenados. A pesar de su carácter social, las normas morales no derivan su aceptabilidad únicamente de lo que la sociedad determine. Las normas morales, en tanto sociales, se caracterizan porque pueden justificarse.

Las normas morales tienen un carácter social porque dependen de las exigencias recíprocas de una comunidad o dependen de una praxis intersubjetiva. En tal sentido, no son normas racionales porque estén fundamentadas en un concepto metafísico de razón, ni puro ni a priori. Para Tugendhat (1988), las normas "son indicaciones generales de acción formuladas en proposiciones" (p. 83) y, más específicamente, son proposiciones de deber ${ }^{4}$. La consecuencia de no seguir las normas es una sanción. A las normas que conllevan una sanción social, esto es, las normas morales, las denomina normas sociales (Tugendhat, 1988, p. 84). Estas tienen un contexto intersubjetivo y por eso cuando no las cumplimos nos hacemos acreedores a una sanción, que se tipifica con la desafección, la censura y el menosprecio, e incluso con la imposición de sanciones externas.

El propósito del autor es mostrar que la norma tiene una cualidad determinada que exige una fundamentación. El modelo de reflexión que utiliza es el de las normas jurídicas, que poseen una cualidad, a saber, la de ser justas, cualidad que, precisamente, exige también de una fundamentación. Pero este es solo el caso especial de las normas jurídicas. En el caso de las normas morales, la validez de la norma reside en que el individuo considere la norma buena para él. Es decir, la norma halla su justificación cuando la concebimos como buena. Por ejemplo,

4 Incluso deber es una palabra normativa débil, mientras que la palabra normativa fuerte es tener que, que indica la obligatoriedad. Tugendhat emplea la palabra deber en lugar de debería porque la primera transmite la obligación categórica o el "tener que" propio de la moral a la que se refiere. 
la norma "no debes hacer sufrir a los demás" posee un predicado de justificación en tanto el individuo la considere como buena para él. Pero hay, además, un segundo paso. No solo se trata de que sea buena para el individuo, sino también para todos los individuos o para un grupo social de individuos que aceptan la norma.

Resumamos lo que hasta aquí dice Tugendhat. El análisis semántico lo condujo al esclarecimiento de los términos bueno, deber y moral. El primer término que aclaró fue el término bueno, poniendo de manifiesto sus usos extramorales, cuyo sentido central es el de preferible. Luego, aclaró los usos morales de la palabra bueno y planteó la posibilidad de que los juicios en los que se usa fueran traducidos a enunciados normativos caracterizados por el término deber. Es decir, que los enunciados con la palabra bueno pueden traducirse a enunciados con la palabra deber. Por ejemplo, el enunciado "no es bueno hacer sufrir a los demás" puede traducirse al enunciado "no debes hacer sufrir a los demás". Tugendhat busca así la justificación de estos enunciados normativos, de manera que la obligatoriedad de estos enunciados radica en que posean una cualidad con la cual puedan valorarse. Esta cualidad es justamente la palabra bueno. La validez de la norma reside, en definitiva, en que el individuo llega a concebirla como buena para él y buena para todos (Tugendhat, 1988, pp. 91-92). Esta es la posición contractualista de Problemas de ética.

Veamos ahora cómo define el contractualismo en Lecciones de ética, en donde Tugendhat sostiene: "me someto a aquellas normas a las que quiero también que se sometan los demás" (Tugendhat, 1997b, p. 81).

\section{2 ÉTICA DEL RESPETO IGUALITARIO}

EN LECCIONES DE ÉTICA, Tugendhat quiere proporcionar una concepción más plausible de la moral que la provista por el contractualismo de Problemas de ética. Para ello, necesita una comprensión del concepto bueno a la que se le vincula un concepto de conciencia moral; a esto denomina precisamente moral del respeto igualitario. A continuación, mostraré en qué consiste esta propuesta morals.

5 Limiñana (2009a) hace una exposición panorámica de la filosofía moral de Tugendhat en la que comienza con Problemas de la ética, continúa con una moral de la seriedad y después considera Ser, verdad, acción. En esta última obra, muestra el autor, Tugendhat reitera la necesidad de justificar los 
Una pregunta fundamental de las Lecciones de ética es si es posible una justificación de las normas morales independientemente de las tradiciones religiosas (Tugendhat, 1997b, p. 15). El primer problema que encuentra es que los juicios morales no se pueden justificar empíricamente. Los demás juicios, excepto los que corresponden a la matemática y a la lógica, son de carácter empírico y su verdad halla su fundamento en la experiencia. Señala además la relación entre los sentimientos o afectos morales y los juicios morales. Se refiere a afectos morales como la indignación, el resentimiento, la culpa y la vergüenza, señalando que los afectos son sentimientos positivos o negativos (placer o displacer), que se construyen sobre un juicio de valor.

Entonces, pasa a precisar su planteamiento. El problema central es la justificación de la moral. Una justificación de la moral no puede ser tradicionalista en el sentido de "tener una autoridad como base de su justificación" (Tugendhat, 1997b, p. 23). Ejemplos de justificación tradicionalista son las creencias que apelan a un mandato divino, a la autoridad o a una tradición que puede ser moral, como los diez mandamientos, o filosófica, cuando se argumenta que así lo dijo un autor. Tugendhat busca, además, un principio de justificación que se diferencie de la justificación trascendental kantiana, porque no se pretende una justificación absoluta. Así, acude a la conciencia moral que, sin tener una base absoluta, posee buenas razones y motivos.

La primera tarea que se propone entonces es clarificar qué es un juicio moral. Desde Aristóteles, reconocemos una proposición enunciativa porque puede ser verdadera o falsa. Los enunciados morales son una clase de enunciado, pero diferente de los empíricos. Tugendhat aclara el significado de los juicios morales con un empleo determinado del grupo de palabras tiene que, no puede, debe y el grupo de palabras bueno y malo. Lo que caracteriza estos juicios es que en la mayoría de los casos hablamos de una necesidad práctica. El ejemplo para el empleo moral del término es "tienes que mantener tu promesa", cuya necesidad no se

juicios morales y afirma que el contractualismo no justifica un sistema moral. "Esto es así porque las sanciones previstas desde la perspectiva del contractualismo tienen un carácter externo, mientras que lo que caracteriza a las normas morales, como con buen criterio apunta Tugendhat, [es que] son eminentemente internas" (Limiñana, 2009a, p. 102). También expone la teoría moral de Lecciones de ética y muestra el desarrollo del autor a partir de cada una de las obras mencionadas. 
fundamenta en un propósito que se quiere alcanzar, sino en que simplemente "hay que hacerlo". Con lo anterior, el autor proporciona un criterio para distinguir qué es un juicio moral.

Señala, justamente, que estas proposiciones universales expresan reglas o normas, que, en general, se definen como "una manera de obrar [que se juzga] como debida" (Tugendhat, 1997b, p. 41). Las reglas prácticas hay que "seguirlas" aunque no obedecen a regularidades de la naturaleza.

Distingue dos tipos diversos de reglas prácticas o normas: las primeras son normas de razón y las otras son reglas denominadas normas sociales. Las normas de razón son las normas que dicta la razón y uno de los mayores exponentes de la comprensión de la moral como un conjunto de normas racionales es Kant. Las normas sociales son normas intersubjetivas en las que nos exigimos unos a otros cómo actuar. Así, cada uno es fuente y destinatario de las normas sociales frente a una comunidad. Las reglas que más ocupan a Tugendhat son las normas sociales, precisamente por su componente sancionatorio.

Una de las características de las normas en general es que se refieren a una sanción. Específicamente, entiende las normas sociales como aquellas que, si no se cumplen, conllevan una sanción social. "Defino el término normas sociales de modo tal que, quien no obra según una norma social, sufre una sanción social” (Tugendhat, 1997b, p. 45). Así distingue tres clases de normas: (1) las normas del derecho en las que la sanción es exterior, como sucede con la prohibición del asesinato o el robo; (2) las normas sociales cuya sanción es interna (Tugendhat, 1997b, p. 45), que el autor explica como una presión social difusa y que pueden ejemplificarse con enunciados como no mentir o no romper las promesas; y (3) las convenciones cuya característica no es la de ser buenas o malas, sino la de ser usuales, como las reglas de urbanidad. La diferencia entre normas sociales y convenciones reside en que mientras Tugendhat caracteriza las convenciones sociales señalando que su fundamento es el rechazo del grupo, con respecto a las normas morales (un tipo particular de normas sociales), su justificación se halla en que el juicio de la acción que corresponde a la norma la evalúa como mala o como buena. Por ello, se compromete con un análisis de los conceptos bueno y malo.

Podemos preguntar qué quiere decir entonces cuando se afirma que algo es bueno o malo. Para responder esta pregunta, Tugendhat intenta mostrar cuáles son los diferentes sentidos de la palabra bueno. Su sentido más general es dar a 
entender que estamos a favor de algo. La palabra bueno ha sido denominada una "palabra pro" (Tugendhat, 1997b, p. 49), lo que quiere decir es que está relacionada con el provecho o con algo favorable y no es una palabra que se opone a algo o que está en contra de algo. Luego de explicar el sentido más general de la palabra bueno, pasa a discutir algunos de sus aspectos como el carácter subjetivo y objetivo o el hecho de que siempre se expresa ante una elección. Pero el aspecto que más le interesa al autor es el de los dos usos que distingue V. Wright y que se emplearon indistintamente en la tradición aristotélica con el nombre de excelencia (areté): en primer lugar, el sentido instrumental y, en segundo lugar, la excelencia técnica. El primer sentido atiende a la utilidad de un objeto, por ejemplo, un reloj que es bueno por dar la hora con precisión; y el segundo sentido se refiere al arte y a las excelencias humanas, como las de un violinista. Este segundo significado le es útil a Tugendhat para aclarar el uso gramaticalmente absoluto de bueno, porque remite a un empleo atributivo de lo excelente. Así, decimos que alguien es bueno "como hombre y como miembro de una comunidad, como compañero o como socio cooperativo" (Tugendhat, 1997b, p. 55). Esto significaría que bueno en este sentido no se refiere primeramente a acciones, sino a personas. Para aclarar este uso atributivo de bueno, Tugendhat se vale de una comparación: así como podemos ser buenos en el desempeño de habilidades, como cantar o tocar el violín, y roles, como ser buenos abogados o maestros, asimismo podemos ser buenos miembros de una comunidad.

Este ser bueno tiene un fundamento emocional. Si ejecuto bien una función y desempeño bien las habilidades requeridas, se puede decir que ello tendría repercusiones positivas para mi sentimiento de autoestima. $\mathrm{Si}$, por el contrario, desempeño mal una habilidad, la reacción puede ser la vergüenza. Como lo define Taylor, "la vergüenza es el sentimiento de pérdida de la autoestima en los ojos de los (posibles) otros" (citado en Tugendhat, 1997b, p. 56). Estas reacciones emocionales también tienen un aspecto moral porque sentimos vergüenza moral cuando fallamos moralmente. A este sentimiento de la vergüenza moral le corresponde el sentimiento de la indignación y reprobación en los demás.

Uno de los conceptos más importantes de las Lecciones de ética es el de conciencia moral, entendida como la comprensión de qué es bueno y de todo lo que se le vincula; se pone de manifiesto cuando todas las premisas trascendentales han desaparecido. Precisamente, los sentimientos morales atienden a la formación 
de la conciencia moral. Tugendhat entiende la formación de la conciencia moral dependiendo de que el individuo se quiera considerar o no como miembro de la comunidad. Este querer o no querer pertenecer a la comunidad moral tiene dos características. Primero, es necesario que el individuo incorpore este querer a su identidad. Segundo, el individuo ha de considerarse miembro de una comunidad de personas que se exigen recíprocamente no violar las normas que constituyen dicha identidad ${ }^{6}$. Este "yo quiero", como perteneciente a la identidad, es la base necesaria del "tener que" o de la obligación moral.

Tugendhat desarrolla, a continuación, su concepción plausible de la moral, esto es, una ética del respeto igualitario. Para ello, se confronta con la filosofía de Kant, dado que tanto Tugendhat como Kant proponen un significado de bueno que sea universalmente válido. La diferencia entre ambos es que mientras Kant busca un significado de bueno que tiene que ser reconocido por todos, Tugendhat presenta un significado de bueno que sea universalmente válido en el sentido de que puede ser reconocido por todos. Vale precisar la diferencia. Esta radica en que para Tugendhat el reconocimiento universal es una posibilidad y no una necesidad. Pero la diferencia más específica es que la concepción kantiana del bien moral, esto es, el imperativo categórico en sus tres formulaciones, se basa en un concepto metafísico de razón, mientras que Tugendhat no busca una fundamentación basada en la razón, sino una justificación basada en una idea plausible del bien que consiste en exigencias recíprocas.

Con todo, este sentido de bueno se encuentra en el contenido de la concepción kantiana expuesta en la llamada segunda fórmula del imperativo categórico, tal y como está consignada en la Fundamentación de la metafísica de las costumbres: "obra de tal modo que uses la humanidad, tanto en tu persona como

6 Tugendhat escribe dos textos sobre identidad personal. "Identidad personal, particular y universal" (2002) y "Particularismo y universalismo" (2002). En el primero, se confronta con la conferencia de Habermas “'Pueden las sociedades complejas desarrollar una identidad racional?”. Aunque Tugendhat expone la tesis de Habermas según la cual las personas "solo pueden llegar a una identidad racional si la colectividad con la que se relacionan es universal” (Tugendhat, 2002, p. 15), señala los errores presentes en ella. Tugendhat quiere mostrar, al distanciarse de la tesis de Habermas, que una "identidad personal lograda tiene que ser al mismo tiempo universal y nacional" (Tugendhat, 2002, p. 15). Esto significa que una identidad nacional, o el nacionalismo, no es necesariamente un descarrío, pues Tugendhat distingue entre un nacionalismo agresivo, como el nazismo, y otro que no lo es. 
en la persona de cualquier otro, siempre como un fin al mismo tiempo y nunca solamente como un medio" (GMS, 429). La traducción que hace Tugendhat es “ ¡No instrumentalices a nadie!”. A esto lo llama una moral del respeto universal.

Esta concepción del respeto universal descansa en una conciencia moral que, vale repetir, se entiende como una comprensión de qué es bueno y de todo lo que se vincula con ello, como los sentimientos morales. Pero el punto aquí es que la conciencia moral nos permite incluir al otro en nuestra reflexión moral; como lo señala el autor, no instrumentalizar al otro ni convertirlo en medio de nuestros intereses. Aquí es importante entender que la conciencia moral no se reduce a una instancia del yo que permite comprender qué sea bueno y malo, sino que se trata de la posibilidad de incluir a una tercera persona en nuestra reflexión sobre qué sea bueno.

Con respecto al problema de cómo entienden las diversas posiciones modernas la justificación de un juicio moral, Tugendhat proporciona una revisión crítica del contractualismo. Cuando se refiere a este, considera que no constituye una moral sino una cuasi-moral. El problema es el modo como se lo entiende, pues el autor distingue dos tipos de contractualismo: el primero es la concepción que Rawls sostiene, según la cual lo moral "se puede entender como el resultado de un contrato ideal que todos pactarían con todos si se encontraran en una situación ideal de igualdad e ignorancia" (Tugendhat, 1997b, p. 72). El segundo tipo es el que remite a la posición expuesta por Mackie en su libro Ethics: Inventing Right and Wrong (1975), en la que se puede hablar de un contrato como base para la fundamentación de la moral, entendiendo el contrato como un convenio implícito. La posición de Mackie apunta al hecho de que los seres humanos están interesados en ponerse de acuerdo para mantener ciertas normas. Casi todas estas reglas concuerdan con la llamada regla de oro: compórtate con respecto a los demás de la manera como deseas que ellos se comporten con respecto a ti. Tugendhat cita a Mackie (1975), quien señala que las reglas son tan fundamentales que incluso una banda de ladrones solo puede existir con dichas normas cooperativas.

Lo anterior plantea un problema que enfrenta el contractualismo: ¿cómo se puede garantizar el cumplimiento de dichas reglas? Precisamente, Tugendhat quiere dejar claro que el contractualismo no puede apelar a la conciencia moral para explicar la razón por la que no se pueden violar las reglas. El contractualismo 
no se basa en un concepto de conciencia moral; se basa en el interés por la cooperación. Si se apelara a la vergüenza o a cualquier sentimiento moral cuando violamos una norma, se apelaría a la conciencia moral y no al interés mismo por la cooperación. Así que, si el contractualismo no puede vincularse al desarrollo de una conciencia moral, tampoco se puede vincular a los demás factores de una moral como el afecto de la indignación o el de la vergüenza. A lo anterior hay que añadir el hecho de que, desde el contractualismo, no se puede juzgar moralmente, pues las reglas son fruto de un acuerdo, y por ello no se posee ninguna concepción sustancial del bien. En suma, el contractualismo no es una moral, sino una cuasi-moral ${ }^{7}$. Sin embargo, lo dicho por Tugendhat sobre el contractualismo implicará una nueva revisión, denominada contractualismo simétrico, que expondré a continuación.

\subsection{CONTRACTUALISMO SIMÉTRICO}

EL TEXTO EL ORIGEN DE LA IGUALDAD NORMATIVA expone la idea de contractualismo simétrico. Para explicarlo, el autor comienza por plantear la pregunta por la justificación de la igualdad y reiterar la pregunta: “ ¿cuál es entonces el origen de nuestra convicción de que no hay legitimidad sin igualdad?” (Tugendhat, 2008, p. 120). En Diálogo en Leticia, Tugendhat había propuesto una solución que ahora le parece insatisfactoria, pero decide presentarla.

Tugendhat explica con dos pasos la primera versión de su solución. El primer paso consiste en derivar consecuencias del concepto de moral. La consecuencia más importante que extrae es que una moral se encuentra justificada cuando todos los miembros de una sociedad moral así lo consideran (Tugendhat, 1999, pp. 21-28). El segundo paso toma en cuenta dos tipos de justificación. El primero es autoritario y el segundo basado en los intereses de la comunidad. Solo se ocupa del segundo (Tugendhat, 1999, pp. 29-35). De este modo, a primera vista pareciera que el autor toma partido por el contractualismo, pues una moral noautoritaria se basa en los intereses de la sociedad moral; pero considerado con

7 Esta explicación de la razón por la que el contractualismo no es una moral se puede encontrar claramente expuesta en Ser, verdad, acción, en el ensayo "Acerca del concepto y de la fundamentación de la moral" (Tugendhat, 1998). 
atención, se distancia del contractualismo ya que su concepción de la moral es que aquello de lo que se dice que es bueno debe ser bueno para todos y el contractualismo no tiene una idea de bondad que regule las relaciones entre quienes pertenecen a la comunidad moral.

Tugendhat encuentra dos problemas en su justificación anterior de la moral con el concepto de igualdad. Primero, ve una cierta circularidad, porque el concepto de igualdad que se busca justificar aparece en la justificación. Segundo, ve la posibilidad de considerar arbitraria la tesis según la cual justificar una moral depende de que esté justificada para todos igualmente.

Por la vía del contractualismo simétrico Tugendhat encuentra "una estructura antropológica fundamental" (Tugendhat, 2008, 123) que le permite responder a la pregunta por la justificación moral. El autor explica que las acciones de los seres humanos tienen su base en la voluntad. La consecuencia de ello es que, si varios seres humanos se comprometen en una acción común, la voluntad de todos integrará esta acción. Considera, al menos, dos formas posibles de esta integración. A la primera la denomina poder y consiste en que solo uno de ellos decide y determina la voluntad del grupo. A esta forma de integración se la puede denominar autoritaria pues hay una concentración unilateral del poder. La otra forma de relación la caracteriza como simétrica. Es la forma opuesta al poder unilateral, pues en ella todos deciden juntos, lo que significa que hay una igualdad en la contribución sobre el actuar.

Como se ve, el trayecto de la teoría moral de Tugendhat y de sus retractaciones lo lleva a exponer varias formas de contractualismo. Aunque algunas veces establece con él una relacion más tensa que otras, en sus diferentes obras nunca pierde de vista que el referente de su teoría moral es el contractualismo.

3. La teoría de la justicia de Tugendhat

Antes De COMENZAR ESTA SECCión, vale aclarar que algunos trabajos sobre Tugendhat se concentran en exponer la filosofía moral del autor -como Limiñana (2009b) - o en vincular la filosofía moral con el análisis conceptual -como Martínez Ferro (2004; 2007)-. En estas obras, no aparece expuesta la relación que se puede establecer entre filosofía política y análisis conceptual. Por otra parte, aunque en los estudios más recientes de Martínez Ferro (2010) y Limiñana (2011) 
sí hay una preocupación por la filosofía política de Tugendhat, no hay referencia alguna al análisis conceptual. El propósito específico de este trabajo es, pues, establecer explícitamente la relación entre el análisis conceptual y la filosofía política de Tugendhat.

Hasta ahora, el hilo conductor de mi texto ha sido exponer el método de Tugendhat, que consiste en la aclaración conceptual de términos como moral, deber y bueno. A continuación, paso a explicar la aclaración conceptual de la justicia y la igualdad. La igualdad para Tugendhat es de carácter normativo, pues todos, dentro de una comunidad moral, somos tanto sujetos de derechos como de obligaciones. $\mathrm{Y}$ en ello, todos somos iguales. No porque seamos iguales corporal, religiosa, racial o sexualmente, ni tampoco porque se abogue por una igualdad en los bienes que poseamos. Esta igualdad se define por el hecho de que todos tenemos los mismos derechos (a la vida, a la educación, a una vivienda digna, a la libertad de palabra, etc.) y que tales derechos son iguales para todos los seres humanos. Por ejemplo, en la Declaración de la Organización de las Naciones Unidas de 1948 se dice que todos tienen estos derechos "sin distinción de cualquier tipo, como raza, color, sexo, lenguaje, religión, opinión política, origen nacional o social" (Asamblea General de la ONU, 1948, art. 2).

En este punto, es pertinente relacionar igualdad y justicia, dado que para Tugendhat la justicia no depende solo del carácter normativo de la igualdad basada en derechos. Para él, la justicia depende del respeto de la igualdad de derechos, pero partiendo del reconocimiento de las desigualdades. Desde esta perspectiva, la justicia y la igualdad tienen un fundamento moral que los convierte en una alternativa al poder unilateral. Para Tugendhat, poder y justicia son dos conceptos opuestos.

En su artículo "Justice", Tugendhat (1994) afirma que su teoría de la justicia es sustantiva y consta de tres pasos, a saber, dos aclaraciones conceptuales y una observación de carácter moral. A continuación, explico estos tres pasos de la teoría de Tugendhat.

La aplicación del método de aclaración conceptual es valiosa en la medida en que no todos los autores que se ocupan de la justicia social se valen de tal método. Tugendhat cita en Lecciones de ética a Rawls y Ackerman para referirse a quienes se ocupan de la justicia social y cuyo lugar común es que no encontramos en ellos un análisis del concepto. Rawls, por ejemplo, opina erróneamente que una 
aclaración del concepto de justicia no contribuye a su comprensión y que simplemente debemos partir de nuestras intuiciones, esto es, la denominada posición original en la que se deciden todas las cuestiones de la justicia política, que se caracteriza por una forma de razonamiento informal y no constituye una prueba. Otra de las razones por la que la posición de Rawls está vinculada a la intuición es porque, en la llamada posición original, el autor recurre al velo de ignorancia que les impide a los participantes de la situación hipotética ideada por Rawls conocer su propia posición, sus objetivos o su situación histórica.

La aclaración conceptual por la que comienza la teoría de la justicia de Tugendhat tiene lugar en el contexto de la justicia distributiva de bienes, cargas y poder. El primer punto que introduce es que, si las personas tienen méritos diferentes, la distribución debe ser diferente (Tugendhat, 1994, p. 4). Como lo afirma en Lecciones, desde Aristóteles es claro que una distribución igualitaria entre desiguales es injusta, sin dejar de considerar que lo que se busca es la igualdad entre las partes.

Tugendhat analiza la justicia distributiva explicando el concepto como una repartición de bienes o males entre varias personas, sea que estas hagan parte de una familia, una empresa común o el Estado. En este tipo de justicia, se considera una repartición de bienes entre los que se encuentran bienes materiales, pero también intangibles, como el poder y los derechos. Lo que quiere señalar Tugendhat es que la justicia distributiva también puede entenderse desde el punto de vista del mérito, pues si la distribución es igualitaria, eso significa que todos merecen lo mismo y si la distribución no es igualitaria, las personas que entran en la distribución pueden merecer más o menos según determinadas razones. La diferencia entre el reparto igualitario y el desigual depende de las razones que justificarían que el mérito sea desigual.

La segunda aclaración conceptual de la teoría de la justicia de Tugendhat se refiere a la diferencia entre dos clases de razones diferentes para una distribución desigual (Tugendhat, 1994, p. 6). El primer tipo de razón lo define como discriminación primaria -creer que alguien tiene más valor intrínsecamente que otro; por ejemplo, la segregación racial-, y el segundo lo define como una discriminación secundaria, aunque de hecho no es propiamente una discriminación, sino una justificación de la distribución desigual.

Tugendhat distingue entonces dos posiciones que se contraponen. La primera es la igualitaria y la segunda es la que denomina aristotélica y consiste en sostener 
que una distribución igual entre desiguales es injusta. Para explicar esta última, propone como ejemplo la distribución de un pastel. Si hay razones para una distribución desigual, esta debe llevarse a cabo. Pensemos en razones como la necesidad: el niño que quiere más pastel puede apelar a que tiene mucha hambre. Sin embargo, también hay razones como los derechos adquiridos, según los cuales el niño puede aducir que su madre le había prometido una mayor porción del pastel. El autor enfatiza que, por una parte, hay razones que justifican el trato desigual mientras que en el caso igualitario se refiere a una regla de distribución más simple.

Esto lo lleva a plantearse la relación entre la concepción igualitaria y la discriminación, pues hay casos en los que puede haber una distribución basada en la discriminación. Al respecto, Tugendhat concluye que la imposibilidad de justificar una discriminación primaria hace que prevalezca una moral igualitaria y explica la distinción entre discriminación primaria y secundaria indicando que la primera se refiere a la existencia de una diferencia previa de valoración de los seres humanos mientras que la segunda es consecuencia de un reparto desigual, pero presupone que todos los seres humanos son iguales. Para ilustrar la discriminación primaria, el autor pone el ejemplo de una creencia como que los blancos tienen mayor valor que los negros. La discriminación secundaria, por otro lado, puede ilustrarse con la distribución de un pastel donde una madre puede presuponer la igualdad de todos sus hijos, pero concederle una mayor porción a quien es el homenajeado en la fiesta de cumpleaños. En tal sentido, Tugendhat se refiere a la discriminación primaria para mostrar que, si se descarta, nos encontramos con una moral igualitaria.

Lo que más le interesa a Tugendhat es el análisis de la justicia desigual justificada, es decir, la discriminación secundaria, y descarta la discriminación primaria, porque carece de justificación, se basa en un prejuicio y, especialmente, porque excluye derechos fundamentales.

El tercer paso en la teoría de la justicia de Tugendhat es el de carácter moral. Ya en la segunda sección de este artículo se expusieron algunos rasgos de su teoría moral. Cabe aclarar, empero, que este paso moral consiste en rechazar la discriminación primaria, lo que equivale a rechazar cualquier apelación a la autoridad religiosa, metafísica o tradicional para justificar cierto tipo de discriminación. En lugar de ello, al justificar las razones de una distribución desigual, se tiene como consecuencia una distribución equitativa entre los participantes 
de la distribución. Vale aclarar aquí, para terminar, que este es justamente uno de los puntos más importantes de la teoría moral de Tugendhat, a saber, su teoría moral no se construye sobre una idea tradicional, autoritaria o metafísica, sino que se construye sobre justificaciones o razones respecto a exigencias recíprocas.

\section{Conclusiones}

LA TESIS DEL TEXTO QUE AQUí PRESENTO, a saber, la existencia de un hilo conductor en algunos aspectos de la filosofía de Ernst Tugendhat que vincula el análisis conceptual, la teoría moral y la teoría de la justicia, se encuentra en el contexto de las múltiples revisiones que hace Tugendhat a sus propuestas filosóficas. Hay quienes encuentran una filosofía unificada, pese a las revisiones que el autor hace de sus propias conclusiones (Eriksen, 2009, p. 4); otros consideran que hay un desarrollo en su pensamiento con variaciones (Martínez, 2007, p. 27) y otros consideran su aspecto sistemático (Bonet, 2013). En este texto, me propuse establecer algunos vínculos y relaciones en la filosofía de Tugendhat a partir de sus propias afirmaciones. Mi opinión es que, aun cuando haya diversidad de intereses en el centro de atención de Tugendhat, no hay un pensamiento desarticulado y en lugar de ello hay un gozne en su reflexión que le podría permitir a sus lectores encontrar una articulación en varios campos a los que se dedicaba como el análisis del lenguaje, la filosofía moral y la filosofía política. El sentido de proponer esta articulación es permitirnos como lectores comprender que los problemas en los que pensaba el autor -como qué es el lenguaje, qué es bueno y qué es la justicia- dan la clave para comprender la unidad de su pensamiento. Y, para ser más especifico, para percatarnos de que las múltiples revisiones críticas por parte de Tugendhat de su pensamiento moral y las continuas retractaciones como su modo de proceder natural, no conducen a la idea de que el pensamiento del autor sea una colcha de retazos. Por el contrario, la revisión de su propio pensamiento tiene como consecuencia la evolución y madurez en sus posiciones sobre la moral entendida como contractualismo. Y por ello, su teoría moral del contractualismo se encuentra representada en el concepto de exigencias recíprocas que permea todo su pensamiento práctico.

Además, mi intención fue exponer algunos aspectos de la teoría de la justicia de Tugendhat y esbozar, aunque muy brevemente, algunas líneas del debate con la teoría de la justicia de Rawls, con quien Tugendhat discute fuertemente 
aspectos de su teoría tanto metodológicos como de contenido y con respecto a quien propone su propia teoría. El debate entre ambos autores se encuentra todavía por explorar y este artículo puede contribuir a iluminarlo teniendo en cuenta el manuscrito inglés denominado "Reply to Tugendhat" ("Respuesta a Tugendhat") ${ }^{8}$ que todavía no sale a la luz. Se trata de la respuesta de Rawls a Tugendhat y a su escrito "Comments on Some Methodological Aspects of Rawls' Theory of Justice"'. Brevemente, las primeras dos secciones del manuscrito tratan sobre el concepto de teoría moral y las dos últimas sobre el concepto de análisis conceptual y su importancia para la teoría de la justicia.

Para mostrar la fecundidad de continuar estudiando esta relación en el futuro, quiero destacar la siguiente cita del manuscrito de Rawls:

Parte de mi dificultad al responder a Tugendhat es que no explica por qué él piensa que el análisis conceptual es tan importante [naturalmente, se refiere a la importancia del análisis conceptual para la teoría de la justicia] [...] Por supuesto, mi posición colapsa si Tugendhat puede dar una explicación de por qué el análisis conceptual es particularmente significativo (pp. 17-19 del manuscrito de Rawls, traducción propia ${ }^{10}$.

8 El artículo se encuentra codificado como HUM 48, box 25, folder 7, Spring 1976, Reply to Tugendhat, y reposa en los archivos personales del Archivo de la Universidad de Harvard. El catálogo de los archivos personales de Rawls puede consultarse en línea en el siguiente enlace: http://oasis.lib. harvard.edu/oasis/deliver/ hua32010. Gracias al equipo de Harvard University Archive accedíal manuscrito de Rawls. Debo el permiso para citar el manuscrito al consentimiento de la familia de Rawls y al de Thomas Scanlon, con quienes estoy muy agradecido.

9 El texto con el que Tugendhat comenta críticamente la Teoría de la justicia (Rawls, 2006) fue leído en un congreso sobre Rawls donde ambos autores estuvieron presentes. El congreso se realizó en abril de 1976, bajo la dirección de Gunter Pätzing en la Fundación Werner-Reimer en Bad Homburg. El texto de Tugendhat se publicó en dos idiomas. Primero en inglés, como versión no corregida en Analyse und Kritik, (1), (1979, pp. 77-89), con el nombre de "Comments on Some Methodological Aspects of Rawls' Theory of Justice”, y más tarde en alemán, en Problem der Ethik como "Bermekungen zu einigen methodischen Aspekten von Rawls' Eine Theorie der Gerechtigkeit". Hay una excelente traducción al castellano del texto como "Observaciones sobre algunos aspectos metodológicos de una Teoría de la justicia de Rawls", a cargo de Jorge Vigil, el cual hace parte del texto Problemas de ética.

10 "Part of my difficulty in replying to Tugendhat is that he does not explain why he thinks conceptual analysis is so important [...]. Of course, if Tugendhat can give an explanation of why conceptual analysis is independently significant, my contention collapses". 


\section{Referencias}

Asamblea General de la ONU. (1948). Declaración Universal de los Derechos Humanos (217 [III] A). París.

Bartolucci, C. (2008). La coscienza linguistica. Ernst Tugendhat tra ontologia e analisi del linguaggio. Perugia: Morlacchi Editore.

Bonet, J. V. (2013). La pregunta más humana de Ernst Tugendhat. València: Universitat de València.

Eriksen, A. (2009). Universalism and Obligation. Is Ernst Tugendhat's Concept of an Emotional Obligation to Universalism Defensible? (Tesis de maestría). Universidad de Oslo, Noruega. Recuperado de: https://www.duo.uio. no/handle/10852/ 24995 ?show=full.

Kant, I. (2010). [GMS]. Fundamentación de la metafisica de las costumbres. (Trad. Roberto Rodríguez Aramayo), Madrid: Alianza Editorial.

Limiñana, P. (2009a). La fundamentación de la moral y la ética del respeto igualitario en el pensamiento de Ernst Tugendhat. Revista Laguna, 24, 97-117.

Limiñana, P. (2009b). La filosofía moral de Ernst Tugendhat. Las Palmas de Gran Canaria: Universidad de las Palmas de Gran Canaria.

Limiñana, P. (2011). Paz, democracia, y derechos humanos. Una perspectiva individualista. Revista Dialética, 2(2), 9-22.

Mackie, J. L. (1975). Ethics: Inventing Right and Wrong. London: Penguin.

Martínez Ferro, H. (2004). El problema de la justificación de la moral en el pensamiento de Ernst Tugendhat (Tesis inédita de maestría). Universidad Nacional de Colombia, Bogotá, Colombia.

Martínez Ferro, H. (2007). Introducción a la filosofía moral de Ernst Tugendhat. Bogotá: Uniediciones.

Martínez Ferro, H. (2010). Los derechos económicos y sociales como mínimos de justicia distributiva. En: Memorias del Cuarto Congreso Nacional y Segundo Internacional de Derecho Constitucional (pp.195-205). Bogotá: Edinar.

Rawls, J. (2006). Teoría de la justicia. (Trad. M. D. González). México/Madrid: Fondo de Cultura Económica.

Rorty, R. (1967). Introduction. En: R. Rorty (Ed.). The Linguistic Turn. Essays in Philosophical Method (pp. 1-39). Chicago: The University of Chicago Press. 
Tugendhat, E. (1976). Vorlesungen zur Einführung in die sprachanalytische Philosophie. Frankfurt: Suhrkamp.

Tugendhat, E. (1982). Traditional and Analytical Philosophy. Lectures on the Philosophy of Language. Cambridge: Cambridge University Press.

Tugendhat, E. (1984). El método analítico. Cuadernos de Filosofia y Letras, VII (1-2), 49-60.

Tugendhat, E. (1988). Problemas de la ética. (Trad. J. Vigil). Barcelona: Editorial Crítica.

Tugendhat, E. (1994). Justice. En: H. Pauer-Studer. (Ed.). Norms, Values, and Society. Vienna Circle Institute Yearbook (vol. 2) (pp. 1-12). Dordrecht: Springer.

Tugendhat, E. (1997a). Propedéutica lógico-semántica. (Trad. G. Hoyos Vásquez). Barcelona: Anthropos.

Tugendhat, E. (1997b). Lecciones de ética. (Trad. L. R. Rabanaque). Barcelona: Gedisa.

Tugendhat, E. (1998). Ser, verdad, acción. Ensayos filosóficos. (Trad. R. H. Santos Ihlau). Barcelona: Gedisa.

Tugendhat, E. (1999). Diálogo en Leticia. (Trad. L. R. Rabanaque). Barcelona: Gedisa.

Tugendhat, E. (2002). Problemas. Barcelona: Gedisa.

Tugendhat, E. (2003). Introducción a la filosofía analítica. Barcelona: Gedisa.

Wittgenstein, L. (1998). The Blue and Brown Books. Oxford: Blackwell.

Wittgenstein, L. (1999). Investigaciones filosóficas. (Trad. A. García Suarez \& U. Moulines). Madrid: Altaya.

Wolf, U. (1999). La filosofía y la cuestión de la vida buena. Madrid: Síntesis.

Zabala, S. (2005). ¿Qué significa pensar tras el giro lingüístico? La filosofía de Ernst Tugendhat. Revista Endoxa: Series filosóficas, 20, 619-637. 